\title{
Dependence of the decoherence of polarization states in phase-damping channels on the frequency spectrum envelope of photons
}

\author{
Yan-Xiao Gong 6 Y Yong-Sheng Zhang 0 Y Yu-Li Dong, Xiao-Ling Niu, Yun-Feng Huang团 and Guang-Can Guo \\ Key Laboratory of Quantum Information, University of Science and Technology of China, \\ CAS, Hefei, 230026, People's Republic of China
}

\begin{abstract}
We consider the decoherence of photons suffering in phase-damping channels. By exploring the evolutions of single-photon polarization states and two-photon polarization-entangled states, we find that different frequency spectrum envelopes of photons induce different decoherence processes. A white frequency spectrum can lead the decoherence to an ideal Markovian process. Some color frequency spectrums can induce asymptotical decoherence, while, some other color frequency spectrums can make coherence vanish periodically with variable revival amplitudes. These behaviors result from the non-Markovian effects on the decoherence process, which may give rise to a revival of coherence after complete decoherence.

PACS numbers: 03.65.Yz, 03.67.Mn, 03.67.Hk
\end{abstract}

\section{INTRODUCTION}

Photons have been widely applied in quantum teleportation 1], quantum dense coding [2], quantum cryptography [3], and quantum computing [4]. Among these, information can be encoded in any of the degrees of freedom (DOF) of photons, such as polarization, frequency, momentum angular, path, or energy time. If we only consider the quantum state in some of the DOF, the other DOF can be regarded as "environment" $[5,6,7,8,19,10,11,12,13,14$. In this context, coupling between different DOF would destroy the coherence of the quantum state considered, leading to decoherence effects, which limit the practical implementation of quantum information processing [15].

In this paper, we consider a non-dissipative coupling between photon frequency and polarization in a birefringent media, resulting in decoherence of polarization due to different group velocities for two orthogonal polarization modes. Coupling between these two DOF has been widely studied in many optical experiments, especially in the field of polarization mode dispersion in optical fibers [16, 17]. Here we simply focus on the phase-damping channels composed of birefringent crystals with fixed optic axes. As this decoherence can be easily controlled by rotating the optic axes or changing the length of birefringent crystals, it has been widely utilized in the experimental research on quantum decoherence dynamics of photons, such as verifying decoherencefree space [5, 6, 7], characterizing entangled mixed states $[8,9,10,11]$. Therefore, it is intuitive and reasonable to investigate the dependence of the decoherence process in this model on the spectrum envelopes of the "environment", namely, the frequency spectrum envelopes (FSEs) of photons.

\footnotetext{
*Electronic address: yxgong@mail.ustc.edu.cn

${ }^{\dagger}$ Electronic address: yshzhang@ustc.edu.cn

‡Electronic address: hyf@ustc.edu.cn
}

The aim of this paper is to address this point by exploring the behaviors of normalized linear entropy [18] of single- and two-photon polarization states, and concurrence [19, 20] of two-photon polarization states. We find that the behaviors of these quantities against the length of birefringent crystals depend on the photon spectrum forms. If the FSE is an ideal white spectrum, the decoherence is Markovian. However, any color spectrums result in non-Markovian decoherence processes. In particular, some FSEs lead to asymptotical decoherence, while, some FSEs induce coherence to vanish periodically with variable revival amplitudes.

Recently, much interest has arisen in the roles of nonMarkovian effects played on quantum states evolution 21, 22, 23, 24, 25, 26, 27, 28, 29, 30, 31, 32]. The "memory effects" of the non-Markovian reservoir can preserve the coherent information of the quantum system during its relaxation time. To show this effect, we obtain the time correlation functions corresponding to different spectrum functions. The correlation function gets a delta-function form when the wave-packet takes the white spectrum, namely the flat spectrum. So in this case, the decoherence is Markovian. If the wave-packet takes nonflat spectrum, the correlation function is a non-trivial function of time, and this behavior can lead a typical non-Markovian effect. Our results then open the door to experimental investigations of the non-Markovian decoherence processes. Moreover, our results on the dynamics of two-photon entanglement, present a possible way to experimental research on entanglement sudden death $[14,33,34,35]$ and recovering entanglement after complete disentanglement.

The rest of our paper is organized as follows. In the next section, we consider the decoherence of singlepolarization states and show the effects of FSEs on the decoherence process by giving several examples of FSEs. In Sec. III we investigate the roles of FSEs played on the evolution of two-photon polarization entangled states, through behaviors of linear entropy and concurrence with some examples of FSEs. In Sec. IV we conclude and 
discuss the experimental feasibility of our results with present photon sources.

\section{DECOHERENCE OF SINGLE-PHOTON POLARIZATION STATES}

We first review the evolution of pure single-photon states in the phase-damping channel based on the calculations in Ref. [6]. An arbitrary single-photon state characterized by its polarization and frequency spectrum can be represented as [6],

$$
|\Psi(0)\rangle=(\alpha|H\rangle+\beta|V\rangle) \otimes \int d \omega f(\omega)|\omega\rangle,
$$

where $|H\rangle(|V\rangle)$ denotes the horizontal (vertical) polarization state with arbitrary complex amplitudes $\alpha$ and $\beta$ satisfying

$$
|\alpha|^{2}+|\beta|^{2}=1,
$$

and $f(\omega)$ is the complex amplitude corresponding to the frequency $\omega$, with the normalization condition,

$$
\int d \omega|f(\omega)|^{2}=\int d \omega F(\omega)=1,
$$

where we use the notation $F(\omega)=|f(\omega)|^{2}$.

Note that for simplicity, polarization and frequency in the initial state we considered is not entangled. The phase-damping channel in our model is composed of a birefringent crystal with a fixed optic axis. Without loss of generality, we set the optic axis in horizontal direction and assume the horizontally polarized photons travel faster than vertically polarized photons, i.e., $n_{H}<n_{V}$. Here $n_{H}\left(n_{V}\right)$ is the index of refraction corresponding to horizontal (vertical) polarization. Then after the photon is transmitted through a birefringent crystal of length $l$, the output state can be expressed as [6],

$$
\begin{aligned}
|\Psi(l)\rangle= & \alpha|H\rangle \otimes \int d \omega f(\omega) e^{i \omega n_{H} l / c}|\omega\rangle \\
& +\beta|V\rangle \otimes \int d \omega f(\omega) e^{i \omega n_{V} l / c}|\omega\rangle .
\end{aligned}
$$

Then we can see that polarization and frequency become entangled. To obtain the polarization state, we trace over the frequency DOF from the density matrix of the output state above, resulting in the output state described as

$$
\rho(l)=\left(\begin{array}{cc}
|\alpha|^{2} & \alpha \beta^{*} \mathcal{F}^{*}(l) \\
\alpha^{*} \beta \mathcal{F}(l) & |\beta|^{2}
\end{array}\right),
$$

where

$$
\mathcal{F}(l)=\int d \omega F(\omega) e^{i \omega \Delta n l / c},
$$

and $\Delta n=n_{V}-n_{H}$. It should be noted that here for simplicity we neglect the variation of the refraction index with $\omega$, since although $n_{H}$ and $n_{V}$ depend on frequency $\omega$, the value of $\Delta n$ does not vary obviously according to $\omega$.

From Eq. (6), we can see $\mathcal{F}(l)$ is the Fourier transform of $F(\omega)$ up to a constant, which depends on the choice of FSE of the photon. As in our decoherence model, the crystal length $l$ is proportional to the time $t$, the function of $\mathcal{F}(l)$ given by Eq. (6) represents the time correlation function in the master equation 36]. If the function is a non-trivial function of length $l$, the decoherence is nonMarkovian, resulting in non-Markovian effects in the evolution of quantum states.

To characterize the decoherence of a single-photon state we employ the normalized linear entropy [18] defined as $S_{L}(\rho) \equiv 2\left[1-\operatorname{Tr}\left(\rho^{2}\right)\right]$ (for pure states $S_{L}=0$ and for mixed states $0<S_{L} \leqslant 1$ ). Then for the output polarization state the linear entropy is given by

$$
S_{L}(l)=2\left[1-\operatorname{Tr}\left(\rho^{2}\right)\right]=4|\alpha|^{2}|\beta|^{2}\left[1-|\mathcal{F}(l)|^{2}\right] .
$$

We can see that the function of linear entropy versus length of the birefringent crystal depends on the FSE. It is necessary to note that if $\alpha=0$ or $\beta=0, S_{L}(l)=0$ independent of $l$, i.e., decoherence does not occur. That is because we set the optic axis horizontal so that the horizontal and vertical polarization states are not affected in such a phase-damping channel.

From the density matrix form given by Eq. (5), we can see that the behavior of the linear entropy is equivalent to that of coherence. In the following part we consider some choices of FSE and present the behaviors of the linear entropy against the crystal length to show the variation of coherence.

\section{A. White spectrum}

We first consider the FSE to be an ideal white spectrum, where $F(\omega)$ does not depend on the frequency. In this case, it is meaningless to consider the normalization condition given by Eq. (3). Usually, the correlation function is assumed to take the form of a delta-function 36, 37], i.e., $\mathcal{F}(l) \sim \delta(l)$. Therefore, complete decoherence occurs for any nonzero $l$. In this context, the decoherence is an ideal Markovian process.

\section{B. Gaussian spectrum}

Let's then choose the FSE to be a Gaussian form written as

$$
F(\omega)=\frac{1}{\Delta \omega \sqrt{\pi}} \exp \left[-\left(\frac{\omega-\omega_{0}}{\Delta \omega}\right)^{2}\right]
$$

where $\omega_{0}$ is the central frequency and $\Delta \omega$ indicates the width of the Gaussian envelope. With this FSE, the cor- 
relation function is found to be

$$
\mathcal{F}(l)=\exp \left[-\left(\frac{\Delta n \Delta \omega l}{2 c}\right)^{2}\right] \exp \left(\frac{i \omega_{0} \Delta n l}{c}\right),
$$

and therefore, the linear entropy becomes

$$
S_{L}(l)=4|\alpha|^{2}|\beta|^{2}\left\{1-\exp \left[-\frac{1}{2}\left(\frac{\Delta n \Delta \omega l}{c}\right)^{2}\right]\right\} .
$$

We can see that $S_{L}$ increases Gaussianly and approaches to maximum asymptotically. Combining the form of the density matrix given by Eq. (5), we can conclude that coherence vanishes asymptotically.

\section{Lorentzian frequency spectrum}

We then consider a Lorentzian frequency spectrum given by

$$
F(\omega)=\frac{\Delta \omega}{\pi} \frac{1}{(\Delta \omega)^{2}+\left(\omega-\omega_{0}\right)^{2}},
$$

where $\omega_{0}$ is the central frequency and $\Delta \omega$ denotes the width of the Lorentzian envelope. Then the correlation function can be expressed as

$$
\mathcal{F}(l)=e^{-\Delta n \Delta \omega l / c} e^{i \Delta n \omega_{0} l / c} .
$$

The linear entropy is therefore

$$
S_{L}(l)=4|\alpha|^{2}|\beta|^{2}\left(1-e^{-2 \Delta n \Delta \omega l / c}\right) .
$$

It is clear that coherence decays exponentially and vanishes asymptotically.

\section{Rectangular spectrum}

A rectangular spectrum is assumed to take the form

$$
F(\omega)= \begin{cases}\frac{1}{2 \Delta \omega} & \left|\omega-\omega_{0}\right| \leqslant \Delta \omega \\ 0 & \left|\omega-\omega_{0}\right|>\Delta \omega\end{cases}
$$

where $\omega_{0}$ is the central frequency and $\Delta \omega$ represents the width of the rectangular envelope. The correlation function becomes

$$
\mathcal{F}(l)=\operatorname{sinc}\left(\frac{\Delta n \Delta \omega l}{c}\right) e^{i \Delta n \omega_{0} l / c},
$$

where the function $\operatorname{sinc} x \equiv \sin x / x$. Therefore, the linear entropy can be expressed as

$$
S_{L}(l)=4|\alpha|^{2}|\beta|^{2}\left[1-\left|\operatorname{sinc}\left(\frac{\Delta n \Delta \omega l}{c}\right)\right|^{2}\right] .
$$

From the above equation and the behavior of the $\operatorname{sinc} x$, we can infer that $S_{L}$ gets to maximum periodically with a damping decrease. This behavior shows a different decoherence process, i.e., coherence vanishes periodically with a damping of its revival amplitude.

\section{E. Multi-peaked spectrum}

Let's first consider an ideal multi-peaked spectrum, called as multi-delta spectrum, described by

$$
F(\omega)=\frac{1}{N} \sum_{j=1}^{N} \delta\left(\omega-\omega_{j}\right)
$$

where $\omega_{j}$ are the peak frequencies. We can see that each peak is a delta spectrum and that if $N \rightarrow \infty$ it is simply the comb spectrum. With this FSE, the correlation function is found to be

$$
\mathcal{F}(l)=\frac{1}{N} \sum_{j=1}^{N} e^{i \Delta n \omega_{j} l / c}
$$

and the linear entropy is expressed as

$$
S_{L}(l)=4|\alpha|^{2}|\beta|^{2}\left[1-\frac{1}{N^{2}}\left|\sum_{j=1}^{N} e^{i \Delta n \omega_{j} l / c}\right|^{2}\right]
$$

From the above equation, it is not difficult to infer that $S_{L}$ can oscillate between 0 and $4|\alpha|^{2}|\beta|^{2}$ (there would be some sub-maximum if $N>2$ ). For simplify, let's analyze the case of $N=2$, and therefore, the linear entropy becomes

$$
S_{L}(l)=4|\alpha|^{2}|\beta|^{2}\left[1-\cos ^{2} \frac{\Delta n\left(\omega_{1}-\omega_{2}\right) l}{2 c}\right] .
$$

The above function shows that $S_{L}$ oscillates between 0 and its maximum. This behavior indicates that coherence can vanish and revival periodically.

Actually, in practical experiments, there is no such ideal spectrum, and the spectrum form at each peak is usually Gaussian, or Lorentzian, or others. Let's first take the Gaussian form for an example. For simplicity, we again assume that there are two peaks, each of which is Gaussian with the same width. The spectrum (called as double-Gaussian spectrum) is written as

$$
\begin{aligned}
F(\omega)=\frac{1}{2 \Delta \omega \sqrt{\pi}} & \left\{\exp \left[-\left(\frac{\omega-\omega_{1}}{\Delta \omega}\right)^{2}\right]\right. \\
& \left.+\exp \left[-\left(\frac{\omega-\omega_{2}}{\Delta \omega}\right)^{2}\right]\right\},
\end{aligned}
$$

where $\omega_{1}$ and $\omega_{2}$ are the two peak frequencies and we assume $\left|\omega_{1}-\omega_{2}\right|=5 \Delta \omega$ so that the two peaks can be considered absolutely separated. Then we can obtain the correlation function as

$$
\begin{aligned}
\mathcal{F}(l)= & \frac{1}{2} \exp \left[-\left(\frac{\Delta n \Delta \omega l}{2 c}\right)^{2}\right] \\
& \times\left[\exp \left(\frac{i \omega_{1} \Delta n l}{c}\right)+\exp \left(\frac{i \omega_{2} \Delta n l}{c}\right)\right],
\end{aligned}
$$




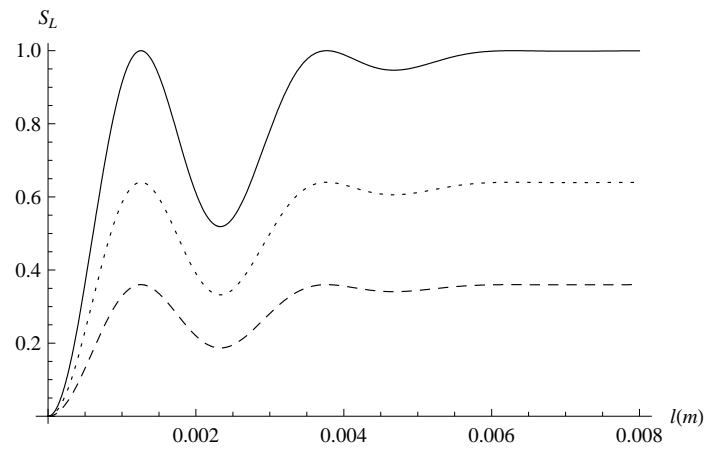

FIG. 1: Linear entropy $S_{L}$ for the polarization state $\alpha|H\rangle+\beta|V\rangle$ with a double-Gaussian frequency spectrum, as a function of crystal length $l$, in the case of $|\alpha|^{2}=0.1$ (dashed line), $|\alpha|^{2}=0.5$ (solid line), and $|\alpha|^{2}=0.8$ (dotted line). Here $|H\rangle(|V\rangle)$ represents the horizontal (vertical) polarization state and $|\alpha|^{2}+|\beta|^{2}=1$.

and the linear entropy as

$$
\begin{aligned}
& S_{L}(l)=4|\alpha|^{2}|\beta|^{2}\{1- \exp \left[-\frac{1}{2}\left(\frac{\Delta n \Delta \omega l}{c}\right)^{2}\right] \\
&\left.\times \cos ^{2} \frac{\Delta n\left(\omega_{1}-\omega_{2}\right) l}{2 c}\right\} .
\end{aligned}
$$

The behaviors of $S_{L}$ against $l$ are plotted in Fig. 1, under the assumption of $\Delta n \Delta \omega / c=500 \mathrm{~m}^{-1}$, in the case of $|\alpha|^{2}=0.1,0.5$, and 0.8 . We can see that coherence vanishes periodically with damped revival amplitudes.

Then we consider an example of double-Lorentzian spectrum given by

$$
\begin{aligned}
F(\omega)= & \frac{\Delta \omega}{2 \pi}\left[\frac{1}{(\Delta \omega)^{2}+\left(\omega-\omega_{1}\right)^{2}}\right. \\
& \left.+\frac{1}{(\Delta \omega)^{2}+\left(\omega-\omega_{2}\right)^{2}}\right],
\end{aligned}
$$

where $\omega_{1}$ and $\omega_{2}$ are the two peak frequencies and we assume $\left|\omega_{1}-\omega_{2}\right|=30 \Delta \omega$ so that the two peaks can be considered absolutely separated. Then the correlation function can be obtained

$$
\mathcal{F}(l)=\frac{1}{2} e^{-\Delta n \Delta \omega l / c}\left(e^{i \Delta n \omega_{1} l / c}+e^{i \Delta n \omega_{2} l / c}\right) .
$$

The linear entropy is therefore

$$
S_{L}(l)=4|\alpha|^{2}|\beta|^{2}\left[1-e^{-2 \Delta n \Delta \omega l / c} \cos ^{2} \frac{\Delta n\left(\omega_{1}-\omega_{2}\right) l}{2 c}\right] .
$$

Fig. 2 shows the behaviors of $S_{L}$ against $l$, under the assumption of $\Delta n \Delta \omega / c=500 \mathrm{~m}^{-1}$, in the case of $|\alpha|^{2}=$ $0.1,0.5$, and 0.8 . We can see that coherence vanishes periodically with damped revival amplitudes.

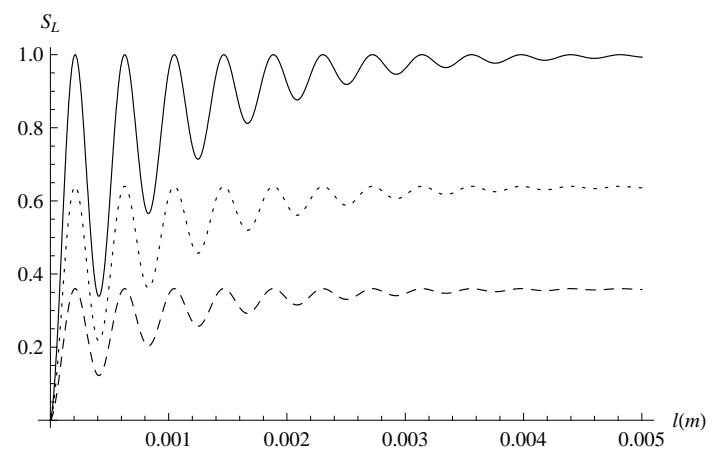

FIG. 2: Linear entropy $S_{L}$ for the polarization state $\alpha|H\rangle+\beta|V\rangle$ with a double-Lorentzian frequency spectrum, as a function of crystal length $l$, in the case of $|\alpha|^{2}=0.1$ (dashed line), $|\alpha|^{2}=0.5$ (solid line), and $|\alpha|^{2}=0.8$ (dotted line). Here $|H\rangle(|V\rangle)$ represents the horizontal (vertical) polarization state and $|\alpha|^{2}+|\beta|^{2}=1$.

\section{DECOHERENCE OF TWO-PHOTON POLARIZATION STATES}

Our analysises above are straightforward to generalize to the decoherence of arbitrary two-photon polarization states. However, here we shall only restrict our analysis to the initial two-photon state given by [6]

$$
\begin{aligned}
|\Phi(0)\rangle= & \left(a|H\rangle_{1}|H\rangle_{2}+b|V\rangle_{1}|V\rangle_{2}\right) \\
& \otimes \int d \omega_{1} d \omega_{2} g\left(\omega_{1}, \omega_{2}\right)\left|\omega_{1}\right\rangle_{1}\left|\omega_{2}\right\rangle_{2},
\end{aligned}
$$

where $a$ and $b$ are arbitrary complex amplitudes satisfying

$$
|a|^{2}+|b|^{2}=1
$$

and $g\left(\omega_{1}, \omega_{2}\right)$ is the complex amplitude corresponding to frequencies $\omega_{1}$ and $\omega_{2}$, with the normalization condition,

$$
\int d \omega_{1} d \omega_{2}\left|g\left(\omega_{1}, \omega_{2}\right)\right|^{2}=\int d \omega_{1} d \omega_{2} G\left(\omega_{1}, \omega_{2}\right)=1,
$$

where the notation $G\left(\omega_{1}, \omega_{2}\right)=\left|g\left(\omega_{1}, \omega_{2}\right)\right|^{2}$. For simplicity, we only consider that only the photon in mode 1 is in

the phase-damping channel the same with that in Sec. II with the other photon in mode 2 free from decoherence. We should note that analogous analysis can be applied to the case of both photons suffering in phase-damping channels. Therefore, after the photon in mode 1 is transmitted through a birefringent crystal of length $l$, the state becomes [ [ ]

$$
\begin{aligned}
|\Phi(l)\rangle= & a|H\rangle_{1}|H\rangle_{2} \\
& \otimes \int d \omega_{1} d \omega_{2} g\left(\omega_{1}, \omega_{2}\right) e^{i \omega_{1} n_{H} l / c}\left|\omega_{1}\right\rangle_{1}\left|\omega_{2}\right\rangle_{2} \\
& +b|V\rangle_{1}|V\rangle_{2} \\
& \otimes \int d \omega_{1} d \omega_{2} g\left(\omega_{1}, \omega_{2}\right) e^{i \omega_{1} n_{V} l / c}\left|\omega_{1}\right\rangle_{1}\left|\omega_{2}\right\rangle_{2} .
\end{aligned}
$$


Tracing over the frequency DOF from the density matrix of the output state above, we can get the output polarization state described as

$$
\rho^{\prime}(l)=\left(\begin{array}{cccc}
|a|^{2} & 0 & 0 & a b^{*} \mathcal{G}^{*}(l) \\
0 & 0 & 0 & 0 \\
0 & 0 & 0 & 0 \\
a^{*} b \mathcal{G}(l) & 0 & 0 & |b|^{2}
\end{array}\right)
$$

where the correlation function is

$$
\mathcal{G}(l)=\int d \omega_{1} d \omega_{2} G\left(\omega_{1}, \omega_{2}\right) e^{i \omega_{1} \Delta n l / c} .
$$

To quantify the mixedness of the state we use the normalized linear entropy [18] given by

$$
S_{L}(l)=\frac{4}{3}\left[1-\operatorname{Tr}\left(\rho^{\prime 2}\right)\right]=\frac{8}{3}|a|^{2}|b|^{2}\left[1-|\mathcal{G}(l)|^{2}\right] .
$$

From the density matrix form of Eq. (31), we can see that the behavior of $S_{L}$ can give the variation of coherence.

To measure the entanglement of a two-photon state $\rho$ we employ the concurrence [19, 20], given by

$$
C(\rho)=\max \left\{0, \sqrt{\lambda_{1}}-\sqrt{\lambda_{2}}-\sqrt{\lambda_{3}}-\sqrt{\lambda_{4}}\right\},
$$

where $\lambda_{i}$ are the eigenvalues of $\rho\left(\sigma_{y} \otimes \sigma_{y}\right) \rho^{*}\left(\sigma_{y} \otimes \sigma_{y}\right)$, in nonincreasing order by magnitude and $\sigma_{y}=\left(\begin{array}{cc}0 & -i \\ i & 0\end{array}\right)$. The case of $C=0$ means no entanglement between the two photons and $0<C \leqslant 1$ corresponds to the existence of entanglement between the two photons. Then for the output state described by Eq. (31), the concurrence is found to be

$$
C(l)=2|a||b||\mathcal{G}(l)| .
$$

Comparing the Eqs. (32), (33) and (35) with Eqs. (6) and (7), we can infer that the effects of FSEs on the decoherence of two-photon states would be similar to that on the decoherence of single-photon states. It is straightforward to study the effects for arbitrary forms of $g\left(\omega_{1}, \omega_{2}\right)$. However, as the purpose of our paper is to show the effects of different types of FSE on the evolution of the polarization states rather than analyze a specific FSE in a practical experiment, it is reasonable to make the following assumptions.

We restrict our analysis to the two-photon polarization states generated by parametric down-conversion, which is widely used in optical experiments. The frequency spectrum takes the form [38]

$$
g\left(\omega_{1}, \omega_{2}\right)=h\left(\omega_{1}\right) h\left(\omega_{2}\right) a_{p}\left(\omega_{p}\right) \varphi\left(\omega_{p}, \omega_{1}-\omega_{2}\right)
$$

with the frequency-anticorrelated relation $\omega_{p}=\omega_{1}+\omega_{2}$, where $h\left(\omega_{1}\right)\left(h\left(\omega_{2}\right)\right)$ represents the transmission function of the optical filter, and $a_{p}\left(\omega_{p}\right)$ describes the pump field spectrum corresponding to the pump frequency $\omega_{p}$, and $\varphi\left(\omega_{p}, \omega_{1}-\omega_{2}\right)$ is the phase-matching function dependent on the size of the non-linear crystal. If the non-linear crystal is thin enough, $\varphi\left(\omega_{p}, \omega_{1}-\omega_{2}\right)$ could be neglected. We make a further simplification by assuming the bandwidth the pump field is very narrow so that $\omega_{p}$ could be considered as a constant. We then have a factorizable spectrum form

$$
g\left(\omega_{1}, \omega_{2}\right) \approx h\left(\omega_{1}\right) h\left(\omega_{2}\right) .
$$

For an ideal white spectrum, again we take the correlation function as a delta-function, i.e., $\mathcal{G}(l) \sim \delta(l)$. In this context, it is clear that both coherence and concurrence vanish for any nonzero $l$, showing an ideal Markovian decoherence process.

For color spectrums, by making $\left|h\left(\omega_{1}\right)\right|^{2}\left(\left|h\left(\omega_{2}\right)\right|^{2}\right)$ take the forms given by Eqs. (8), (11), (14), (21) and (24) (normalization constants may be needed to satisfy Eq. (29)), we give some examples as follows,

a Gaussian spectrum centered at $\omega_{p} / 2$ :

$$
\begin{aligned}
G\left(\omega_{1}, \omega_{2}\right) \approx & \sqrt{\frac{2}{\pi}} \frac{1}{\Delta \omega} \exp \left[-2\left(\frac{\omega_{1}-\omega_{p} / 2}{\Delta \omega}\right)^{2}\right] \\
& \times \delta\left(\omega_{p}-\omega_{1}-\omega_{2}\right)
\end{aligned}
$$

a Lorentzian spectrum centered at $\omega_{p} / 2$ :

$$
G\left(\omega_{1}, \omega_{2}\right) \approx \frac{2}{\pi} \frac{(\Delta \omega)^{3} \delta\left(\omega_{p}-\omega_{1}-\omega_{2}\right)}{\left[(\Delta \omega)^{2}+\left(\omega_{1}-\omega_{P} / 2\right)^{2}\right]^{2}},
$$

a rectangular spectrum centered at $\omega_{p} / 2$ :

$$
G\left(\omega_{1}, \omega_{2}\right) \approx \begin{cases}\frac{\delta\left(\omega_{p}-\omega_{1}-\omega_{2}\right)}{2 \Delta \omega} & \left|\omega_{1}-\omega_{p} / 2\right| \leqslant \Delta \omega \\ 0 & \left|\omega_{1}-\omega_{p} / 2\right|>\Delta \omega\end{cases}
$$

a double-Gaussian spectrum centered at $\left(\omega_{p}-5 \Delta \omega\right) / 2$ and $\left(\omega_{p}+5 \Delta \omega\right) / 2$ :

$$
\begin{aligned}
G\left(\omega_{1}, \omega_{2}\right) & \approx \frac{1}{\sqrt{2 \pi} \Delta \omega} \delta\left(\omega_{p}-\omega_{1}-\omega_{2}\right) \\
& \times\left\{\exp \left[-2\left(\frac{\omega_{1}-\left(\omega_{p}-5 \Delta \omega\right) / 2}{\Delta \omega}\right)^{2}\right]\right. \\
& \left.+\exp \left[-2\left(\frac{\omega_{1}-\left(\omega_{p}+5 \Delta \omega\right) / 2}{\Delta \omega}\right)^{2}\right]\right\} .
\end{aligned}
$$

a double-Lorentzian spectrum centered at $\left(\omega_{p}-30 \Delta \omega\right) / 2$ and $\left(\omega_{p}+30 \Delta \omega\right) / 2$ :

$$
\begin{aligned}
G\left(\omega_{1}, \omega_{2}\right) & \approx \frac{1}{\pi}(\Delta \omega)^{3} \delta\left(\omega_{p}-\omega_{1}-\omega_{2}\right) \\
& \times\left\{\left[(\Delta \omega)^{2}+\left(\omega_{1}-\omega_{p} / 2+15 \Delta \omega\right)^{2}\right]^{-2}\right. \\
& \left.+\left[(\Delta \omega)^{2}+\left(\omega_{1}-\omega_{p} / 2-15 \Delta \omega\right)^{2}\right]^{-2}\right\} .
\end{aligned}
$$

Through the analogous analysises in Sec. II] we can obtain the correlation function $\mathcal{G}(l)$, the linear entropy $S_{L}(l)$ and the concurrence $C(l)$, and investigate the different decoherence processes. For simplicity, we plotted 


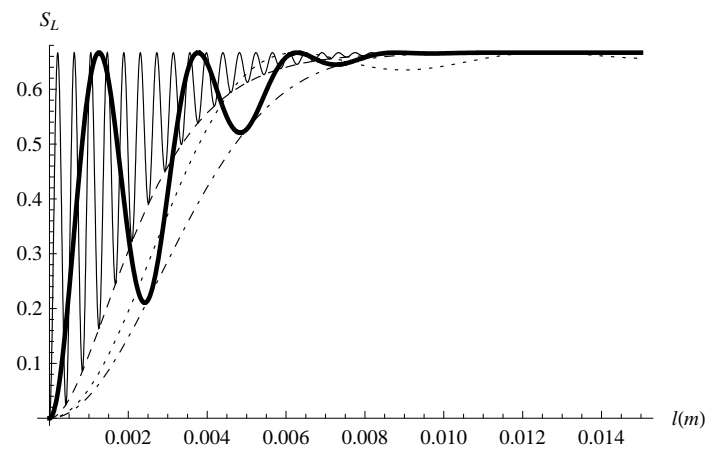

(a)

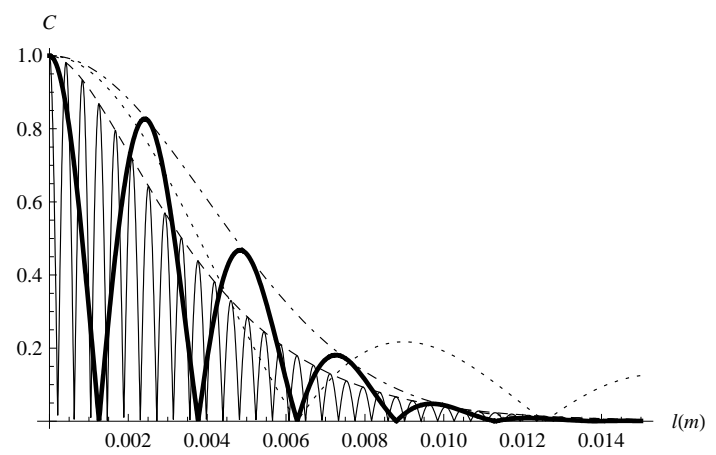

(b)

FIG. 3: (a) Linear entropy $S_{L}(l)$ (b) Concurrence $C(l)$ of the initial polarization state as a function of crystal length $l$ with a Gaussian spectrum (dot-dashed line), a Lorentzian spectrum (dashed line), a rectangular spectrum (dotted line), a doubleGaussian spectrum (thick solid line) and a double-Lorentzian spectrum (thin solid line).

the behaviors of linear entropy (see Fig. 3(a) and concurrence (see Fig. 3(b) of the specific initial polarization state $(|H H\rangle+|V V\rangle) / \sqrt{2}$ against the crystal length $l$ in the case of the four FSEs shown above (again we set $\left.\Delta n \Delta \omega / c=500 m^{-1}\right)$.

We can see that coherence and concurrence vanish asymptotically in the case of the Gaussian spectrum and the Lorentzian spectrum, while vanish periodically with damped revival amplitudes in the case of the rectangular spectrum, the double-Gaussian spectrum and the doubleLorentzian spectrum.

\section{CONCLUSIONS AND DISCUSSIONS}

We have considered the effects of photon spectrum forms on the decoherence process in phase-damping channels. We have found that the behavior of coherence depends on the choice of FSE. We have also given some examples of the forms of FSE to show different behaviors. An ideal white spectrum gives rise to an ideal Markovian decoherence process, while, color spectrums result in non-Markovian correlation functions, inducing non-Markovian effects on the decoherence process. Ex- plicitly, among our examples, against the crystal length, coherence was found to vanish asymptotically in the case of a Gaussian spectrum and a Lorentzian spectrum, while periodically with variable revival amplitudes in the case of a rectangular spectrum and a multi-peaked spectrum.

We would like to discuss the experimental feasibility of our results briefly. In our paper, we only gave theoretical analysises with several ideal choices of FSE, however, analogous analysises can appply to any practical spectrum forms. Furthermore, photons with various FSEs have been experimentally realized. The spectrum filter usually used may restrict the FSE to a Gaussian form [6]. Quantum dot [39, 40] and fluorescence [41, 42, 43, 44] based sources may generate photons with a Lorentzian spectrum. Keller et al. [45] have demonstrated the productions of Gaussian, rectangular, and double-peaked wave functions of photons emitted from Raman pumped single ions trapped in a cavity by manipulating the pulse pump. The case of Double-Lorentzian functions may find possible applications to the case of photonic bandgap. There have been a few experimental reports on shaping wave packets of entangled photons from parametric down-conversion [38, 46, 47, 48]. Moreover, it should be noted that some of us have ever observed the coherence revivals in the phase-damping channels by restricting the photon spectrum using filters with a rectangular transmission function (see Appendix in Ref. [49]), and that during our preparation of this paper, we became aware that, by shaping the spectrum of photons with a Fabry-Perot Cavity, coherence revivals of single- and twophoton polarization states in the phase-damping channels had been observed [50].

Besides the Refs. [38, 46, 47, 48], the spectral influences on the photon correlations or fourth-order interference [51, 52, 53, 54, 55, 56] have been extensively studied. Recently, the decoherence of photon pairs from parametric down-conversion have been experimentally studied and characterized in terms of fluctuations of pump laser [57, 58]. Our approach would be possible to find applications with these studies. We hope our work can stimulate more investigations on the characteristics of photon spectrum distributions. For instance, further results beyond the studies on polarization mode dispersion in optical fibers [59, 60] would be obtained if different types of FSE could be considered.

\section{Acknowledgments}

Y.X.G. thanks Chuan-Feng Li, Jin-Shi Xu and ChaoYang Lu for helpful discussions. This work was funded by National Fundamental Research Program (Grant No. 2006CB921907), National Natural Science Foundation of China (Grants No. 10674127, No. 60621064 and No. 10774139), Innovation Funds from Chinese Academy of Sciences, Program for New Century Excellent Talents in University, A Foundation for the Author of National Excellent Doctoral Dissertation of PR China 
(grant 200729).

[1] D. Bouwmeester, J.-W. Pan, K. Mattle, M. Eibl, H. Weinfurter, and A. Zeilinger, Nature (London) 390, 575 (1997).

[2] K. Mattle, H. Weinfurter, P. G. Kwiat, and A. Zeilinger, Phys. Rev. Lett. 76, 4656 (1996).

[3] N. Gisin, G. Ribordy, W. Tittel, and H. Zbinden, Rev. Mod. Phys. 74, 145 (2002).

[4] P. Kok, W. J. Munro, K. Nemoto, T. C. Ralph, J. P. Dowling, and G. J. Milburn, Rev. Mod. Phys. 79, 135 (2007).

[5] P. G. Kwiat, A. J. Berglund, J. B. Altepeter, and A. G. White, Science 290, 498 (2000).

[6] A. J. Berglund, quant-ph/0010001 (2000).

[7] J. B. Altepeter, P. G. Hadley, S. M. Wendelken, A. J. Berglund, and P. G. Kwiat, Phys. Rev. Lett. 92, 147901 (2004).

[8] A. G. White, D. F. V. James, W. J. Munro, and P. G. Kwiat, Phys. Rev. A 65, 012301 (2001).

[9] R. T. Thew and W. J. Munro, Phys. Rev. A 64, 022320 (2001).

[10] Y.-S. Zhang, Y.-F. Huang, C.-F. Li, and G.-C. Guo, Phys. Rev. A 66, 062315 (2002).

[11] N. A. Peters, J. B. Altepeter, D. Branning, E. R. Jeffrey, T.-C. Wei, and P. G. Kwiat, Phys. Rev. Lett. 92, 133601 (2004).

[12] M. Barbieri, F. De Martini, G. Di Nepi, and P. Mataloni, Phys. Rev. Lett. 92, 177901 (2004).

[13] G. Puentes, A. Aiello, D. Voigt, and J. P. Woerdman, Phys. Rev. A 75, 032319 (2007).

[14] M. P. Almeida, F. de Melo, M. Hor-Meyll, A. Salles, S. P. Walborn, P. H. S. Ribeiro, and L. Davidovich, Science 316, 579 (2007).

[15] W. H. Zurek, Phys. Today 44, 36 (1991).

[16] J. P. Gordon and H. Kogelnik, Proc. Natl. Acad. Sci. U.S.A. 97, 4541 (2000).

[17] L. E. Nelson and R. M. Jopson, J. Opt. Fiber. Commun. Rep. 1, 312 (2004).

[18] S. Bose and V. Vedral, Phys. Rev. A 61, 040101 (2000).

[19] W. K. Wootters, Phys. Rev. Lett. 80, 2245 (1998).

[20] V. Coffman, J. Kundu, and W. K. Wootters, Phys. Rev. A 61, 052306 (2000).

[21] D. Ahn, J. Lee, M. S. Kim, and S. W. Hwang, Phys. Rev. A 66, 012302 (2002).

[22] J. Lee, I. Kim, D. Ahn, H. McAneney, and M. S. Kim, Phys. Rev. A 70, 024301 (2004).

[23] S. Shresta, C. Anastopoulos, A. Dragulescu, and B. L. Hu, Phys. Rev. A 71, 022109 (2005).

[24] M. Ban, S. Kitajima, and F. Shibata, J. Phys. A 38, 7161 (2005).

[25] M. Ban, J. Phys. A 39, 1927 (2006).

[26] I. Glendinning, M. Jakob, and M. Noelle, quantph/0604030 (2006).

[27] S. Maniscalco, S. Olivares, and M. G. A. Paris, Phys. Rev. A 75, 062119 (2007).

[28] B. Bellomo, R. LoFranco, and G. Compagno, Phys. Rev. Lett. 99, 160502 (2007).

[29] K.-L. Liu and H.-S. Goan, Phys. Rev. A 76, 022312 (2007).
[30] J. Dajka, M. Mierzejewski, and J. Łuczka, Phys. Rev. A 77, 042316 (2008).

[31] J. Piilo, S. Maniscalco, K. Härkönen, and K.-A. Suominen, Phys. Rev. Lett. 100, 180402 (2008).

[32] B. Bellomo, R. L. Franco, and G. Compagno, Phys. Rev. A 77, 032342 (2008).

[33] T. Yu and J. H. Eberly, Phys. Rev. Lett. 93, 140404 (2004).

[34] T. Yu and J. H. Eberly, Opt. Commun. 264, 393 (2006).

[35] T. Yu and J. H. Eberly, Phys. Rev. Lett. 97, 140403 (2006).

[36] C. W. Gardiner and P. Zoller, Quantum Noise (SpringerVerlag, New York, 2000).

[37] G. A. Prataviera, S. S. Mizrahi, V. V. Dodonov, and J. R. Brinati, Phys. Rev. A 60, 4045 (1999).

[38] A. Pe'er, B. Dayan, A. A. Friesem, and Y. Silberberg, Phys. Rev. Lett. 94, 073601 (2005).

[39] E. Moreau, I. Robert, J. M. Gérard, I. Abram, L. Manin, and V. Thierry-Mieg, Appl. Phys. Lett. 79, 2865 (2001).

[40] C. Santori, D. Fattal, G. S. S. Vučković, Jelena, and Y. Yamamoto, Nature (London) 419, 594 (2002).

[41] C. Brunel, B. Lounis, P. Tamarat, and M. Orrit, Phys. Rev. Lett. 83, 2722 (1999).

[42] B. Lounis and W. E. Moerner, Nature (London) 407, 491 (2000).

[43] G. Wrigge, I. Gerhardt, J. Hwang, G. Zumofen, and V. Sandoghdar1, Nat. Phys. 4, 60 (2008).

[44] A. N. Vamivakas, Y. Zhao, C.-Y. Lu, and M. Atatüre, arXiv:0806.3707.

[45] M. Keller, B. Lange, K. Hayasaka, W. Lange, and H. Walther, Nature (London) 431, 1075 (2004).

[46] M. Bellini, F. Marin, S. Viciani, A. Zavatta, and F. T. Arecchi, Phys. Rev. Lett. 90, 043602 (2003).

[47] S. Viciani, A. Zavatta, and M. Bellini, Phys. Rev. A 69, 053801 (2004).

[48] S.-Y. Baek, O. Kwon, and Y.-H. Kim, Phys. Rev. A 77, 013829 (2008).

[49] Y.-S. Zhang, Y.-F. Huang, C.-F. Li, and G.-C. Guo, quant-ph/0206166 (2002).

[50] J.-S. Xu, C.-F. Li, M. Gong, X.-B. Zou, G. Chen, and G.-C. Guo, submitted.

[51] M. H. Rubin, D. N. Klyshko, Y. H. Shih, and A. V. Sergienko, Phys. Rev. A 50, 5122 (1994).

[52] T. E. Keller and M. H. Rubin, Phys. Rev. A 56, 1534 (1997).

[53] W. P. Grice and I. A. Walmsley, Phys. Rev. A 56, 1627 (1997).

[54] W. P. Grice, R. Erdmann, I. A. Walmsley, and D. Branning, Phys. Rev. A 57, R2289 (1998).

[55] R. Andrews, A. T. Joseph, E. R. Pike, and S. Sarkar, J. Opt. B: Quantum Semiclassical Opt. 7, S480 (2005).

[56] P. P. Rohde, T. C. Ralph, and M. A. Nielsen, Phys. Rev. A 72, 052332 (2005).

[57] S. Cialdi, F. Castelli, I. Boscolo, and M. G. A. Paris, Appl. Opt. 47, 1831 (2008).

[58] S. Cialdi, F. Castelli, and M. G. A. Paris, arXiv:0802.3131.

[59] Y.-S. Zhang and G.-C. Guo, Chin. Phys. Lett. 23, 2129 
(2006).

[60] P. S. Y. Poon and C. K. Law, Phys. Rev. A 77, 032330

(2008). 